kann aber der Franck-Condon-Faktor für die Anregung der ersten Quanten u. U. sehr klein werden.

Der Zerfall $\mathrm{CH}_{3} \mathrm{OH}^{+} \rightarrow \mathrm{CH}_{2} \mathrm{OH}^{+}+\mathrm{H}$ beginnt nach Chupka bei 11,67 eV. In der Koinzidenzkurve hört die Bildung von $\mathrm{CH}_{3} \mathrm{OH}^{+}$im Einklang mit der geringen Besetzungsdichte, die bei der Photoionisation erreicht wird, oberhalb $11,4 \mathrm{eV}$ auf, bevor die Frag. mentbildung in Gang kommt. Wilmenius und Lindholm sehen ebenfalls ein abruptes Abbrechen der Muttermasse im Zerfallsdiagramm am Einsatzpunkt der Bruchstückbildung. Die Koinzidenzkurve des $\mathrm{CH}_{2} \mathrm{OH}^{+}$folgt in ihrem Verhalten der Gegenfeldkurve; Ionen im zweiten elektronischen Zustand zerfallen in $\mathrm{CH}_{2} \mathrm{OH}+\mathrm{H}$.

\section{Ameisensäure}

$\mathrm{HCOOH}$ ist ein relativ einfaches, unsymmetrisches Molekül. Das AP des Ions wurde von WATANABE $^{30} \mathrm{zu} 11,05 \mathrm{eV}$ bestimmt. Dieser Wert wurde hier als Eichpunkt von Gegenfeldkurve und Koinzidenzkurven, Abb. 12, benutzt. Bei 12,00 $\pm 0,05 \mathrm{eV}$ beginnt ein zweiter, bei $13,91 \pm 0,05 \mathrm{eV}$ ein dritter elektronischer Zustand des Ions. In der Koinzidenzkurve hört die Produktion von $\mathrm{HCOOH}^{+}$mit dem Ausklingen des Grundzustandes auf; das AP des Bruchstückes $\mathrm{HCOO}^{+}$, nach MARINER und BleAKNEY ${ }^{32}$ bei $12,1 \pm 0,1 \mathrm{eV}$, fällt zusammen mit dem Beginn des zweiten elektronischen Zustands. Bei $12,9 \pm 0,25 \mathrm{eV}$ hört auch die Bruchstückbildung von $\mathrm{HCOO}^{+}$auf, nach Mariner und Bleakney beginnt bei $12,8 \mathrm{eV}$ der Zerfall

$$
\mathrm{HCOOH}^{+} \rightarrow \mathrm{OH}+\mathrm{HCO}^{+} \text {. }
$$

Ich danke Herrn Prof. Dr. O. Osberghaus für die Anregung zu dieser Arbeit und besonders Herrn Dr. B. BREHM für viele klärende Diskussionen.

32 T. Mariner u. W. Bleakney, Phys. Rev. 72, 792 [1947].

\title{
Zur Thermodynamik flüssiger unvollständig dissoziierter binärer Elektrolytmischungen
}

\author{
K.-H. DÜCKER \\ Institut für Physikalische Chemie der Rheinisch-Westfälischen Technischen Hochschule Aachen
}

(Z. Naturforsch. 25 a, 1071-1080 [1970]; eingegangen am 4. April 1970)

\begin{abstract}
Für eine Elektrolytmischung aus zwei Komponenten, deren Moleküle in einer Stufe dissoziieren und die ein gemeinsames Ion (Kation oder Anion) besitzen, werden die chemischen Potentiale der Komponenten und der in der Mischung vorhandenen Teilchenarten zueinander in Beziehung gesetzt. In Analogie zur „idealen assoziierten Mischung“ oder „idealen vollständig dissoziierten Mischung“ wird die „ideale unvollständig dissoziierte Mischung“ definiert. Die Aktivitätskoeffizienten der Komponenten dieser idealen Mischung haben im gesamten Konzentrationsbereich den Wert 1. Die Betrachtung des Verdampfungsgleichgewichtes liefert Ausdrücke zur Berechnung der Aktivitätskoeffizienten aus Partialdampfdruckmessungen. Einige Spezialfälle werden erörtert.
\end{abstract}

\section{Einführung}

Wir betrachten eine flüssige Mischung aus zwei Komponenten 1 und 2. Die Moleküle der beiden Komponenten können teilweise in einer Stufe in geladene Teilchen dissoziiert sein, wobei insgesamt drei verschiedene Ionenarten entstehen. Ein Molekül der Komponente 1 bzw. 2 zerfällt in die Kationen $A^{z_{a}}$ und Anionen $B^{z_{b}}$ bzw. Kationen $C^{z_{c}}$ und die gleichen Anionen ${ }^{1} B^{z_{b}} . z_{a}, z_{b}$ und $z_{c}$ sind die Ladungszahlen der Dissoziationsprodukte $A, B$ und $C$. Die

Sonderdruckanforderungen an Dr. K.-H. DücKER, Institut für Physikalische Chemie der Techn. Hochschule Aachen, D-5100 Aachen, Templergraben 59.
Komponente 1 bzw. 2 mit den Zerfallszahlen $\nu_{a}$ und $v_{b}$ bzw. $v_{c}$ und $v_{b}{ }^{*}$ ihrer Ionen $A^{z_{a}}, B^{z_{b}}$ bzw. $C^{z_{c}}$ und $B^{z_{b}}$ hat die Formel $A_{v a} B_{v b}$ bzw. $C_{v_{c}} B_{v b^{*}}$. Es liegen also in der Mischung die Dissoziationsgleichgewichte

$$
A_{v_{a}} B_{v_{b}} \rightleftharpoons v_{a} A^{z_{a}}+v_{a} B^{z_{b}} \quad \text { (Komp. 1) }
$$

und $\quad C_{v_{c}} B_{v_{b}^{*}} \rightleftharpoons v_{c} C^{z_{c}}+v_{b}^{*} B^{z_{b}} \quad$ (Komp. 2)

vor, die bei der Komponente 1 durch den Dissoziationsgrad $\alpha_{1}$ und bei der Komponente 2 durch den Dissoziationsgrad $\alpha_{2}$ charakterisiert sind. Wegen der Elektroneutralität gelten die Beziehungen:

1 Die Annahme gleicher Anionen ist willkürlich. Die folgenden Überlegungen lassen sich natürlich auch für Systeme mit gleichen Kationen durchführen. 


$$
\begin{aligned}
& \boldsymbol{v}_{a} z_{a}+\boldsymbol{v}_{b} z_{b}=0, \\
& \boldsymbol{v}_{c} z_{c}+\boldsymbol{v}_{b}{ }^{*} z_{b}=0 .
\end{aligned}
$$

Als makroskopische Variable unserer flüssigen Mischung dienen die thermodynamische Temperatur $T$, der Druck $P$ und der stöchiometrische Molenbruch $x$ der Komponente 1. Die Dissoziationsgrade $\alpha_{1}$ und $\alpha_{2}$ der beiden Komponenten 1 und 2 hängen von diesen Zustandsvariablen ab. Da die vorgegebene Mischphase nur zwei Komponenten enthält, hat der stöchiometrische Molenbruch der Komponente 2 den Wert $1-x$. Bei $x=1$ bzw. $x=0$ liegt die reine Komponente 1 bzw. 2 vor. Beispiele für solche Systeme sind Mischungen flüssiger organischer Säuren oder teilweise dissoziierte Elektrolytschmelzen mit einem gemeinsamen Ion.

\section{Komponenten und Teilchenarten}

Die oben beschriebene Mischung enthält die Ionen $A^{z_{a}}, B^{z_{b}}, C^{z_{c}}$ sowie die neutralen Teilchenarten $A_{v_{a}} B_{v_{b}}$ und $C_{v_{c}} B_{v_{b}}^{*}$. Zu ihnen gehören die wahren Molenbrüche $x_{a}, x_{b}, x_{c}, x_{a b}$ und $x_{c b}$. Sie sind gegeben durch die Zusammenhänge

$$
\begin{aligned}
x_{a} & =v_{a} \alpha_{1} x / N, \\
x_{b} & =\left[v_{b} \alpha_{1} x+v_{b}^{*} \alpha_{2}(1-x)\right] / N, \\
x_{c} & =v_{c} \alpha_{2}(1-x) / N, \\
x_{a b} & =\left(1-\alpha_{1}\right) x / N \\
x_{c b} & =\left(1-\alpha_{2}\right)(1-x) / N,
\end{aligned}
$$

und

wobei

$N \equiv 1+\left(v_{2}-1\right) \alpha_{2}+\left[\left(v_{1}-1\right) \alpha_{1}-\left(v_{2}-1\right) \alpha_{2}\right] x$

mit

und

$$
\begin{aligned}
& v_{1} \equiv v_{a}+v_{b} \\
& \nu_{2} \equiv v_{c}+v_{b}^{*}
\end{aligned}
$$

gesetzt ist.

Die reinen Komponenten 1 und 2 besitzen die Dissoziationsgrade $\alpha_{1}{ }^{0}$ und $\alpha_{2}{ }^{0}$, die Funktionen der Temperatur $T$ und des Druckes $P$ sind. Die wahren Molenbrüche $x_{a}^{0}, x_{b}^{0}$ und $x_{a b}^{0}$ der in der reinen Komponente 1 existierenden Teilchenarten ergeben sich aus den Gln. (3), (4) und (6) beim Grenzübergang $x \rightarrow 1\left(\alpha_{1} \rightarrow a_{1}{ }^{0}\right)$ für feste Temperatur $T$ und festen Druck $P$ zu:

$$
\begin{aligned}
& x_{a}^{0}=\lim _{x \rightarrow 1} x_{a}=\frac{v_{a} a_{1}{ }^{0}}{1+\left(v_{1}-1\right) a_{1}{ }^{0}}, \\
& x_{b}^{0}=\lim _{x \rightarrow 1} x_{b}=\frac{v_{b} a_{1}{ }^{0}}{1+\left(v_{1}-1\right) a_{1}{ }^{0}}
\end{aligned}
$$

und $\quad x_{a b}^{0}=\lim _{x \rightarrow 1} x_{a b}=\frac{1-a_{1}{ }^{0}}{1+\left(v_{1}-1\right) a_{1}{ }^{0}}$.

Entsprechend findet man für die wahren Molenbrüche $x_{c}^{0}, x_{b}^{0 *}$ und $x_{c b}^{0}$ der in der reinen Komponente 2 vorhandenen Teilchenarten nach den Gln. (4), (5) und (7) beim Grenzübergang $x \rightarrow 0$ $\left(\alpha_{2} \rightarrow \alpha_{2}{ }^{0}\right)$ für konstante Temperatur $T$ und konstanten Druck $P$ :

$$
\begin{aligned}
& x_{c}^{0}=\lim _{x \rightarrow 0} x_{c}=\frac{v_{c} a_{2}{ }^{0}}{1+\left(v_{2}-1\right) a_{2}^{0}}, \\
& x_{b}^{0 *}=\lim _{x \rightarrow 0} x_{b}=\frac{v_{b}^{*} a_{2}{ }^{0}}{1+\left(v_{2}-1\right) a_{2}{ }^{0}}
\end{aligned}
$$

und

$$
x_{c b}^{0}=\lim _{x \rightarrow 0} x_{c b}=\frac{1-a_{2}{ }^{0}}{1+\left(v_{2}-1\right) a_{2}{ }^{0}} .
$$

Wenn eine Komponente undissoziiert ist, liegt ein Sonderfall unseres Systems vor. Wir haben dann die Mischung eines teilweise dissoziierten Elektrolyten mit einem neutralen Stoff, also ein System, das gewöhnlich im Rahmen der Elektrolytlösungen behandelt wird. Dissoziiert zum Beispiel die Komponente 1 nicht, erhält man mit $\alpha_{1}=0$ für die wahren Molenbrüche der jetzt vorhandenen Teilchenarten aus den Gln. (3) bis (10) :

$$
\begin{aligned}
& x_{a}=0, \\
& x_{b}=v_{b}^{*} \alpha_{2}(1-x) / M, \\
& x_{c}=v_{c} \alpha_{2}(1-x) / M, \\
& x_{a b}=x / M
\end{aligned}
$$$$
\text { und } \quad x_{c b}=\left(1-\alpha_{2}\right)(1-x) / M
$$$$
\text { mit } \quad M \equiv 1+\left(v_{2}-1\right) \alpha_{2}(1-x) \text {. }
$$

Der Grenzübergang $x \rightarrow 1$ liefert in diesem Spezialfall der undissoziierten Komponente 1 mit $\alpha_{1}=\alpha_{1}{ }^{0}$ $=0$ nach (11), (12) und (13) :

$$
x_{a}^{0}=0, \quad x_{b}^{0}=0 \quad \text { und } \quad x_{a b}^{0}=1, \quad(23 \mathrm{a}, \mathrm{b}, \mathrm{c})
$$

während für den anderen Grenzfall $x \rightarrow 0$ die Beziehungen (14), (15) und (16) erhalten bleiben.

Sind beide Komponenten undissoziiert, liegt eine Mischung von Nichtelektrolyten vor, die eine besondere Gruppe der Mischphasen bildet.

\section{Chemische Potentiale}

Für die Freie Enthalpie $G$ einer Mischung aus den Molmengen $n_{k}$ der Stoffe $k$ mit den chemischen Potentialen $\mu_{k}$ gilt allgemein ${ }^{2}$ :

2 R. HAase, Thermodynamik der Mischphasen, Verlag Springer, Berlin 1956. 


$$
G=\sum_{k} n_{k} \mu_{k}
$$

Hierbei ist es gleichgültig, ob man als Stoffe $k$ die Komponenten oder die Teilchenarten wählt. Bedeuten $n_{1}$ und $n_{2}$ bzw. $\mu_{1}$ und $\mu_{2}$ die stöchiometrischen Molmengen bzw. die chemischen Potentiale der Komponenten 1 und 2 und $n_{a}, n_{b}, n_{c}, n_{a b}$ und $n_{c b}$ bzw. $\mu_{a}, \mu_{b}, \mu_{c}, \mu_{a b}$ und $\mu_{c b}$ die wahren Molmengen bzw. die chemischen Potentiale der Teilchenarten $A^{z_{a}}, B^{z_{b}}, C^{z_{c}}, A_{v_{a}} B_{v_{b}}$ und $C_{v_{c}} B_{v_{b}{ }^{*}}$, folgt aus (24) :

$$
\begin{aligned}
G & =n_{1} \mu_{1}+n_{2} \mu_{2} \\
& =n_{a} \mu_{a}+n_{b} \mu_{b}+n_{c} \mu_{c}+n_{a b} \mu_{a b}+n_{c b} \mu_{c b} .
\end{aligned}
$$

Zwischen den wahren und den stöchiometrischen Molmengen bestehen die Zusammenhänge:

$$
\begin{aligned}
& n_{a}=v_{a} \alpha_{1} n_{1}, \\
& n_{b}=v_{b} \alpha_{1} n_{1}+v_{b}^{*} \alpha_{2} n_{2}, \\
& n_{c}=v_{c} \alpha_{2} n_{2}, \\
& n_{a b}=\left(1-\alpha_{1}\right) n_{1}
\end{aligned}
$$

und $\quad n_{c b}=\left(1-\alpha_{2}\right) n_{2}$.

Die Gl. (25) ergibt mit den Umrechnungen (26) und den allgemeinen Gleichgewichtsbedingungen für die Dissoziationsgleichgewichte (1 a) und (1 b) ${ }^{2}$

$$
\mu_{a b}=v_{a} \mu_{a}+v_{b} \mu_{b}
$$

und

$$
\mu_{c b}=v_{c} \mu_{c}+v_{b}^{*} \mu_{b}
$$

die Beziehung

$$
n_{1} \mu_{1}+n_{2} \mu_{2}=n_{1} \mu_{a b}+n_{2} \mu_{c b},
$$

woraus durch Koeffizientenvergleich unter Verwendung von (27 a) und (27 b) folgt:

$$
\begin{aligned}
& \mu_{1}=\mu_{a b}=v_{a} \mu_{a}+v_{b} \mu_{b}, \\
& \mu_{2}=\mu_{c b}=v_{c} \mu_{c}+v_{b}{ }^{*} \mu_{b} .
\end{aligned}
$$

Bezeichnet man mit $\mu_{01}$ bzw. $\mu_{02}$ die chemischen Potentiale der reinen flüssigen Komponenten 1 und 2, mit $\mu_{a}^{0}$ bzw. $\mu_{b}^{0}$ bzw. $\mu_{a b}^{0}$ die chemischen Potentiale der Teilchenarten $A^{z_{a}}$ bzw. $B^{z_{b}}$ bzw. $A_{v_{a}} B_{v_{b}}$ der reinen flüssigen Komponente 1 und mit $\mu_{c}^{0}$ bzw. $\mu_{b}^{0 *}$ bzw. $\mu_{c b}^{0}$ die chemischen Potentiale der Teilchenarten $C^{z_{c}}$ bzw. $B^{z_{b}}$ bzw. $C_{v_{c}} B_{v_{b}^{*}}$ der reinen flüssigen Kom. ponente ${ }^{3} 2$ und definiert sie für unser System durch

$$
\mu_{01} \equiv \lim _{x \rightarrow 1} \mu_{1}, \quad \quad \mu_{02} \equiv \lim _{x \rightarrow 0} \mu_{2}, \quad(30 \mathrm{a}, \mathrm{b})
$$

\footnotetext{
${ }^{3}$ Die reinen Komponenten müssen bei denselben Werten der Temperatur $T$ und des Druckes $P$ vorliegen wie die Mischung.
}

$$
\begin{aligned}
& \mu_{a}^{0} \equiv \lim _{x \rightarrow 1} \mu_{a}, \quad \quad \mu_{b}^{0} \equiv \lim _{x \rightarrow 1} \mu_{b}, \quad(30 \mathrm{c}, \mathrm{d}) \\
& \mu_{a b}^{0} \equiv \lim _{x \rightarrow 1} \mu_{a b}, \quad \quad \mu_{c}^{0} \equiv \lim _{x \rightarrow 0} \mu_{c}, \quad(30 \text { e, f }) \\
& \mu_{b}^{0 *} \equiv \lim _{x \rightarrow 0} \mu_{b} \quad \text { und } \quad \mu_{c b}^{0} \equiv \lim _{x \rightarrow 0} \mu_{c b}, \quad(30 \mathrm{~g}, \mathrm{~h})
\end{aligned}
$$

erhält man aus (29a) und (29b) zusammen mit $(30 \mathrm{a}),(30 \mathrm{~b}),(30 \mathrm{e})$ und $(30 \mathrm{~h})$ :

$$
\mu_{01}=\lim _{x \rightarrow 1} \mu_{1}=\lim _{x \rightarrow 1} \mu_{a b}=\mu_{a b}^{0}
$$

und $\quad \mu_{02}=\lim _{x \rightarrow 0} \mu_{2}=\lim _{x \rightarrow 0} \mu_{c b}=\mu_{c b}^{0}$.

Beachtung von $(27 \mathrm{a}),(27 \mathrm{~b}),(30 \mathrm{c}),(30 \mathrm{~d})$, $(30 \mathrm{f})$ und (30 g) führt aus (31 a) und (31 b) zu:

$$
\begin{aligned}
& \mu_{01}=v_{a} \mu_{a}^{0}+v_{b} \mu_{b}^{0}=\mu_{a b}^{0}, \\
& \mu_{02}=v_{c} \mu_{c}^{0}+v_{b}^{*} \mu_{b}^{*}=\mu_{a b}^{0} .
\end{aligned}
$$

\section{Aktivität}

Wir wollen nun die dimensionslose Funktion $a_{i}$ der Komponente $i$ durch die Definition

$$
\ln a_{i} \equiv \frac{\mu_{i}-\mu_{0 i}}{R T} \quad(i=1,2)
$$

einführen und sie die Aktivität der Komponente $i$ nennen. $\mu_{i}$ ist das chemische Potential der Komponente $i, \mu_{0 i}$ das chemische Potential der reinen flüssigen Komponente $i$ bei denselben Werten der Temperatur und des Druckes $P$ und $R$ die Gaskonstante. Durch Einsetzen von (29a) und (32a) sowie von $(29 \mathrm{~b})$ und $(32 \mathrm{~b})$ in (33) ergibt sich:

$$
\begin{aligned}
\ln a_{1} & =\frac{1}{R T}\left[v_{a}\left(\mu_{a}-\mu_{a}^{0}\right)+v_{b}\left(\mu_{b}-\mu_{b}^{0}\right)\right] \\
& =\frac{1}{R T}\left[\mu_{a b}-\mu_{a b}^{0}\right], \\
\ln a_{2} & =\frac{1}{R T}\left[v_{c}\left(\mu_{c}-\mu_{c}^{0}\right)+v_{b}^{*}\left(\mu_{b}-\mu_{b}^{0 *}\right)\right] \\
& =\frac{1}{R T}\left[\mu_{c b}-\mu_{a b}^{0}\right] .
\end{aligned}
$$

Die Aktivitäten $a_{1}$ und $a_{2}$ stellen meßbare Größen dar, die von der Temperatur $T$, vom Druck $P$ und von der Zusammensetzung $x$ abhängen. Sie sind nach der Gibbs-Duhemschen Gleichung bei fester Temperatur $T$ und festem Druck $P$ durch

$$
x\left(\frac{\partial \ln a_{1}}{\partial x}\right)_{T, P}+(1-x)\left(\frac{\partial \ln a_{2}}{\partial x}\right)_{T, P}=0
$$

miteinander verknüpft.

Die Gln. (29a), (29 b), (31 a), (31 b), (32 a), $(32 \mathrm{~b}),(34 \mathrm{a})$ und $(34 \mathrm{~b})$ gelten für beliebigen Dis- 
soziationsgrad $\alpha_{i}$ bzw. $\alpha_{i}{ }^{0}\left(i=1,2 ; 0 \leqq \alpha_{i}, \alpha_{i}{ }^{0} \leqq 1\right)$ unter der Voraussetzung des Dissoziationsgleichgewichtes $(27 \mathrm{a})$ und $(27 \mathrm{~b})$. Läßt sich das chemische Potential $\mu_{j}$ aller in der Mischung vorhandenen geladenen und ungeladenen Teilchenarten $A^{z_{a}}, B^{z_{b}}, C^{z_{c}}$, $A_{v_{a}} B_{v_{b}}$ und $C_{v_{c}} B_{v_{b}^{*}}{ }^{*}$ durch die Beziehung

$$
\mu_{j}=\mu_{0 j}+R T \ln x_{j} \quad(j=a, b, c, a b, c b)
$$

im Bereich von $x_{j}=0$ bis $x_{j}=1$ beschreiben, liegt eine ideale teilweise dissoziierte Mischung vor. $\mu_{j}$ ist das chemische Potential der Teilchenart $j$ in der Mischung, $\mu_{0 j}$ das chemische Potential der reinen hypothetischen Teilchenart $j$ bei fester Temperatur $T$ und festem Druck $P, R$ die Gaskonstante, $T$ die thermodynamische Temperatur und $x_{j}$ der wahre Molenbruch der Teilchenart $j$. Eine solche ideale unvollständig dissoziierte Mischung stellt ein Gegenstück zur idealen assoziierten Mischung bei Nichtelektrolyten dar. Sie ist bei hinreichender Ähnlichkeit der einzelnen Partikel denkbar ${ }^{4}$. Für die verschiedenen Teilchen unserer Mischung gilt dann:

$$
\begin{aligned}
& \mu_{a}=\mu_{0 a}+R T \ln x_{a}, \\
& \mu_{b}=\mu_{0 b}+R T \ln x_{b}, \\
& \mu_{c}=\mu_{0 c}+R T \ln x_{c}, \\
& \mu_{a b}=\mu_{0 a b}+R T \ln x_{a b}
\end{aligned}
$$

und

$$
\mu_{c b}=\mu_{0 c b}+R T \ln x_{c b} .
$$

$\mu_{0 a}, \mu_{0 b}$ und $\mu_{0 c}$ sind die chemischen Potentiale der reinen (hypothetischen) flüssigen Ionen $A^{z_{a}}, B^{z_{b}}$ und $C^{z_{c}}, \mu_{0 a b}$ und $\mu_{0 c b}$ die der reinen (hypothetischen) neutralen Moleküle $A_{v_{a}} B_{v_{b}}$ und $C_{v} B_{v_{b e}^{*}}$.

Der Übergang zur reinen Komponente $1(x=1)$ bzw. $2(x=0)$ liefert mit (11), (12), (13), (30 c), $(30 \mathrm{~d})$ und $(30 \mathrm{e})$ aus $(37 \mathrm{a}),(37 \mathrm{~b})$ und $(37 \mathrm{~d})$ :

$$
\begin{aligned}
& \mu_{a}^{0}=\mu_{0 c}+R T \ln x_{c}^{0}, \\
& \mu_{b}^{0}=\mu_{0 b}+R T \ln x_{b}^{0}
\end{aligned}
$$

und

$$
\mu_{a b}^{0}=\mu_{0 a b}+R T \ln x_{a b}^{0},
$$

bzw. mit (14), (15), (16), (30 f), (30 g) und $(30 \mathrm{~h})$ aus $(37 \mathrm{~b}),(37 \mathrm{c})$ und $(37 \mathrm{e})$

$$
\begin{aligned}
& \mu_{c}^{0}=\mu_{0 c}+R T \ln x_{c}^{0}, \\
& \mu_{b}^{0 *}=\mu_{0 b}+R T \ln x_{b}^{0 *}
\end{aligned}
$$

und

$$
\mu_{c b}^{0}=\mu_{0 c b}+R T \ln x_{c b}^{0} .
$$

Die Differenz aus den entsprechenden Gln. (37) und (38) führt zu

$$
\begin{aligned}
& \mu_{a}-\mu_{a}^{0}=R T \ln x_{a} / x_{a}^{0}, \\
& \mu_{b}-\mu_{b}^{0}=R T \ln x_{b} / x_{b}^{0}, \\
& \mu_{a b}-\mu_{a b}^{0}=R T \ln x_{a b} / x_{a b}^{0}, \\
& \mu_{c}-\mu_{c}^{0}=R T \ln x_{c} / x_{c}^{0}, \\
& \mu_{b}-\mu_{b}^{0 *}=R T \ln x_{b} / x_{b}^{0 *}
\end{aligned}
$$

und

$$
\mu_{c b}-\mu_{a b}^{0}=R T \ln x_{c b} / x_{a b}^{0} .
$$

Führt man die Beziehungen (39a) und (39 b) oder (39 c) in den Ausdruck (34 a) ein, ergibt sich mit

$$
a_{1}=a_{1} \text { id }
$$

der Zusammenhang

$$
a_{1}^{\mathrm{id}}=\left(x_{a} / x_{a}^{0}\right)^{v_{a}}\left(x_{b} / x_{b}^{0}\right)^{v_{b}}
$$

oder

$$
a_{1}{ }^{\mathrm{id}}=x_{a b} / x_{a b}^{0} .
$$

Analog findet man aus (34 b), (39 d), (39e) und (39 f) mit

$$
a_{2}=a_{2}{ }^{\mathrm{id}}
$$

die Beziehung

$$
a_{2}{ }^{\mathrm{id}}=\left(x_{c} / x_{c}^{0}\right)^{v_{c}} \cdot\left(x_{b} / x_{b}^{0 *}\right)^{v_{b}^{*}}
$$

oder

$$
a_{2}{ }^{\mathrm{id}}=x_{c b} / x_{c b}^{0} \text {. }
$$

Zur Umrechnung der in den Gln. (41) und (42) auftretenden wahren Molenbrüche in die stöchiometrischen Molenbrüche setzt man die Ausdrücke (3), (4), (11) und (12) in (41 a) bzw. (4), (5), (14) und (15) in (41 b) ein und erhält:

$$
\begin{gathered}
a_{1}^{\mathrm{id}}=\left[\alpha_{1} x\right]^{v_{a}}\left[\alpha_{1} x+\frac{v_{b}^{*}}{v_{b}} \alpha_{2}(1-x)\right]^{v_{b}}\left[\frac{1+\left(v_{1}-1\right) a_{1}{ }^{0}}{a_{1}{ }^{0}\left[1+\left(v_{2}-1\right) a_{2}+\left(\left(v_{1}-1\right) a_{1}-\left(v_{2}-1\right) \alpha_{2}\right) x\right]}\right]^{\nu_{1}} \\
\text { bzw. } \left.\quad a_{2}{ }^{\mathrm{id}}=\left[\alpha_{2}(1-x)\right]^{v_{c}}\left[\alpha_{2}(1-x)+\frac{v_{b}}{v_{b}{ }^{*}} \alpha_{1} x\right]^{*}\left[\frac{1+\left(v_{2}-1\right) a_{2}{ }^{0}}{a_{2}{ }^{0}\left[1+\left(v_{2}-1\right) a_{2}+\left(\left(v_{1}-1\right) a_{1}-\left(v_{2}-1\right) a_{2}\right) x\right]}\right]\right]^{\nu_{2}},
\end{gathered}
$$

${ }^{4}$ HOENEN ${ }^{5}$ hat bei seinen thermodynamischen Betrachtungen von Schmelzgleichgewichten dissoziierender Stoffe im Bereich hoher Verdünnung einer Komponente ebenfalls die wahren Molenbrüche aller vorhandenen Teilchenarten benutzt und „ideale“ Verhältnisse angenommen.

5 P. H. J. Hoenen, Z. Physik. Chem. 83, 513 [1913]. 
wobei auch die Gln. 8), (9) und (10) benutzt wurden. Analog erhält man durch Einsetzen von (6) und (13) in (42a) bzw. von (7) und (16) in $(42 \mathrm{~b})$ :

$a_{1}{ }^{\text {id }}=\frac{\left(1-a_{1}\right)\left[1+\left(v_{1}-1\right) a_{1}^{0}\right] x}{\left(1-a_{1}^{0}\right)\left[1+\left(v_{2}-1\right) a_{2}+\left(\left(v_{1}-1\right) a_{1}-\left(v_{2}-1\right) a_{2}\right) x\right]}$

bzw.

$a_{2}{ }^{\mathrm{id}}=\frac{\left(1-a_{2}\right)\left[1+\left(v_{2}-1\right) a_{2}^{0}\right](1-x)}{\left(1-a_{2}^{0}\right)\left[1+\left(v_{2}-1\right) a_{2}+\left(\left(v_{1}-1\right) a_{1}-\left(v_{2}-1\right) a_{2}\right) x\right]}$

Man erkennt den symmetrischen Charakter der Ausdrücke (44a) und (44 b) sowie (45a) und (45 b). Eine unvollständig dissoziierte ideale Elektrolytmischung wird durch die Beziehungen (44) und (45) beschrieben. Die Größen auf den rechten Seiten dieser Gleichungen sind prinzipiell meßbar. Sie gestatten es, eine teilweise dissoziierte Elektrolytmischung aus zwei Komponenten mit einem gemeinsamen Ion zu charakterisieren. Im Gegensatz zur idealen vollständig dissoziierten flüssigen Elektrolytmischung, bei der die Aktivitäten $a_{1}$ und $a_{2}$ nur Funktionen der Zusammensetzung $x$ sind ${ }^{6}$, hängen die Aktivitäten $a_{1}$ und $a_{2}$ der Komponenten 1 und 2 bei der unvollständig dissoziierten Elektrolytmischung außerdem noch über die Dissoziationsgrade $a_{1}, a_{1}^{0}, a_{2}$ und $\alpha_{2}^{0}$ von der Temperatur $T$ und vom Druck $P$ ab.

Bei vollständiger Dissoziation $\left(\alpha_{1}=\alpha_{1}{ }^{0}=\alpha_{2}=\alpha_{2}{ }^{0}\right.$ $=1$ ) folgen aus Gln. (44a) und $(44 \mathrm{~b})$ die von HAASE ${ }^{6,7}$ abgeleiteten Beziehungen für ideale vollständig dissoziierte Elektrolytschmelzen, wobei im ersten Fall die Funktion $\Psi_{i}(i=1,2)$ benutzt wurde, die mit der Aktivität $a_{i}$ durch

verknüpft ist.

$$
\ln a_{i}=\Psi_{i}
$$

Für den oben erwähnten Sonderfall der undissoziierten Komponente 1 [Mischung eines Elektrolyten (2) mit einem neutralen Stoff (1)] ergibt sich aus (20), (22), (23 c) und (42 a) bei der Komponente 1:

$$
a_{1}^{\mathrm{id}}=\frac{x}{1+\left(v_{2}-1\right) a_{2}(1-x)},
$$

eine Beziehung, die auch aus (45a) mit $\alpha_{1}=\alpha_{1}{ }^{0}=0$ folgt. Für die Komponente 2 erhält man unter diesen Bedingungen aus (14), (15), (18), (19) und $(41 \mathrm{~b})$ oder aus $(16),(21)$ und $(42 \mathrm{~b})$ :

$$
a_{2}^{\text {id }}=\left[\frac{a_{2}\left(1+\left(v_{2}-1\right) a_{2}^{0}\right)(1-x)}{a_{2}^{0}\left(1+\left(v_{2}-1\right) a_{2}(1-x)\right)}\right]^{\nu_{2}},
$$

oder $\quad a_{2}^{\mathrm{id}}=\frac{\left(1-a_{2}\right)\left(1+\left(v_{2}-1\right) a_{2}^{0}\right)(1-x)}{\left(1-a_{2}^{0}\right)\left(1+\left(v_{2}-1\right) a_{2}(1-x)\right)}$.

Die Zusammenhänge (46b) und (46c) findet man ebenfalls aus $(44 \mathrm{~b})$ oder $(45 \mathrm{~b})$ mit $\alpha_{1}=\alpha_{1}{ }^{0}=0$.

Im folgenden sollen nun beispielhaft für vier Spezialtypen von Mischungen aus zwei Komponenten 1 und 2 die Aktivitäten $a_{1}$ und $a_{2}$ berechnet werden.

I. Liegt ein besonders einfaches System aus zwei Komponenten vom Typ $\mathrm{AB}+\mathrm{CB}$ (zum Beispiel $\mathrm{HClO}_{4}+\mathrm{NaClO}_{4}$ mit einer vollständig dissoziierten Komponente oder $\mathrm{HCOOH}+\mathrm{Cl}_{3} \mathrm{CCOOH}$ mit zwei unvollständig dissoziierten Komponenten und gemeinsamem Kation) vor, dessen Moleküle in jeweils ein Kation und ein gemeinsames Anion zerfallen, gilt gemäß (9) und (10) :

$$
\begin{aligned}
v_{a}=v_{b} & =v_{c}=v_{b}{ }^{*}=1, \\
v_{1} & =v_{2}=2 .
\end{aligned}
$$

Man erhält dann für dieses Beispiel aus (44a), (44, b) und (47) :

$$
\begin{aligned}
a_{1}{ }^{\mathrm{id}}= & \alpha_{1} x\left(a_{1} x+\alpha_{2}(1-x)\right) \\
& \cdot\left[\frac{1+a_{1}^{0}}{a_{1}{ }^{0}\left(1+a_{2}+\left(a_{1}-a_{2}\right) x\right)}\right]^{2} \equiv K_{1} \mathrm{I}, \\
a_{2}{ }^{\mathrm{id}}= & \alpha_{2}(1-x)\left(\alpha_{2}(1-x)+\alpha_{1} x\right) \\
& \cdot\left[\frac{1+a_{2}^{0}}{a_{2}{ }^{0}\left(1+a_{2}+\left(a_{1}-a_{2}\right) x\right)}\right]^{2} \equiv K_{2}{ }^{\mathrm{I}} .
\end{aligned}
$$

Bei vollständiger Dissoziation gelten mit

$$
\alpha_{1}=\alpha_{1}{ }^{0}=\alpha_{2}=\alpha_{2}{ }^{0}=1
$$

nach (48 a) und (48b) die Beziehungen

$$
\begin{array}{ll}
a_{1}{ }^{\mathrm{id}}=x \\
\text { und } & a_{2}{ }^{\mathrm{id}}=(1-x) .
\end{array}
$$

II. Dissoziieren die beiden Komponenten des Typs $\mathrm{A}_{2} \mathrm{~B}_{2}+\mathrm{C}_{2} \mathrm{~B}_{2}$ in jeweils zwei Kationen und zwei gemeinsame Anionen, ergibt sich mit (9) und (10) :

$$
\begin{aligned}
v_{a}=v_{b} & =v_{c}=v_{b}^{*}=2, \\
v_{1} & =v_{2}=4 .
\end{aligned}
$$

Man findet hier aus (44a) und (44 b) mit (50)

$$
\mathrm{a}_{1}{ }^{\mathrm{id}}=\left[\alpha_{1} x\left(\alpha_{1} x+\alpha_{2}(1-x)\right)\right]^{2}\left[\frac{1+3 a_{1}{ }^{0}}{a_{1}{ }^{0}\left(1+3 a_{2}+\left(a_{1}-a_{2}\right) 3 x\right)}\right]^{4} \equiv K_{1} \mathrm{II}
$$

6 R. HaAse, Z. Physik. Chem. N.F. 63, 95 [1969].

7 R. HAase, J. Phys. Chem. 73, 1160 [1969]. 
und

$$
a_{2}^{\mathrm{id}}=\left[\alpha_{2}(1-x)\left(\alpha_{2}(1-x)+\alpha_{1} x\right)\right]^{2}\left[\frac{1+3 a_{2}{ }^{0}}{\alpha_{2}{ }^{0}\left(1+3 \alpha_{2}+\left(\alpha_{1}-\alpha_{2}\right) 3 x\right)}\right]^{4} \equiv K_{2}{ }^{\mathrm{II}},
$$

woraus für vollständige Dissoziation $\left(\alpha_{1}=\alpha_{1}{ }^{0}=\alpha_{2}=\alpha_{2}{ }^{0}=1\right)$ folgt:

$$
a_{1}{ }^{\mathrm{id}}=x^{2}, \quad a_{2}{ }^{\mathrm{id}}=(1-x)^{2} .
$$

III. Bilden die Moleküle der Stoffe 1 und 2 vom Typ $\mathrm{A}_{2} \mathrm{~B}+\mathrm{C}_{2} \mathrm{~B}$ (zum Beispiel $\mathrm{Na}_{2} \mathrm{SO}_{4}+\mathrm{K}_{2} \mathrm{SO}_{4}$ ) beide zwei Kationen und ein gemeinsames Anion, gilt nach (9) und (10) :

$$
v_{a}=v_{c}=2, \quad v_{b}=v_{b}{ }^{*}=1, \quad v_{1}=v_{2}=3,
$$

und (44a) und (44b) liefern mit (53) :

und

$$
\begin{aligned}
& a_{1}{ }^{\mathrm{id}}=\left[\alpha_{1} x\right]^{2}\left[\alpha_{1} x+\alpha_{2}(1-x)\right]\left[\frac{1+2 \alpha_{1}{ }^{0}}{\alpha_{1}{ }^{0}\left(1+2 \alpha_{2}+\left(\alpha_{1}-\alpha_{2}\right) 2 x\right)}\right]^{3} \equiv K_{1}{ }^{\mathrm{III}} \\
& a_{2}{ }^{\mathrm{id}}=\left[\alpha_{2}(1-x)\right]^{2}\left[\alpha_{2}(1-x)+\alpha_{2} x\right]\left[\frac{1+2 \alpha_{2}{ }^{0}}{a_{2}{ }^{0}\left(1+2 \alpha_{2}+\left(\alpha_{1}-\alpha_{2}\right) 2 x\right)}\right]^{3} \equiv K_{2}{ }^{\mathrm{III}} .
\end{aligned}
$$

Bei vollständiger Dissoziation $\left(\alpha_{1}=\alpha_{1}{ }^{0}=\alpha_{2}=\alpha_{2}{ }^{0}=1\right)$ erhält man daraus :

$$
a_{1}{ }^{\mathrm{id}}=x^{2}, \quad a_{2}{ }^{\mathrm{id}}=(1-x)^{2} . \quad(55 \mathrm{a}, \mathrm{b})
$$

IV. Liefert die Dissoziation der Komponenten 1 und 2 des Typs $\mathrm{AB}_{2}+\mathrm{CB}_{2}$ [z. B. Magnesiumstearat
+ Calciumstearat: $\left.\mathrm{Mg}\left(\mathrm{C}_{18} \mathrm{H}_{35} \mathrm{O}_{2}\right)_{2}+\mathrm{Ca}\left(\mathrm{C}_{18} \mathrm{H}_{35} \mathrm{O}_{2}\right)_{2}\right]$ unserer Mischung aus einem Molekül ein Kation und zwei gemeinsame Anionen, führen (9) und (10) $\mathrm{mit}$

$$
\boldsymbol{v}_{a}=\boldsymbol{v}_{c}=1, \quad v_{b}=\boldsymbol{v}_{b}^{*}=2 \text { und } \boldsymbol{v}_{\mathbf{1}}=\boldsymbol{v}_{\mathbf{2}}=3
$$

sowie $(44 \mathrm{a}),(44 \mathrm{~b})$ und $(56) \mathrm{zu}$

und

$$
\begin{aligned}
& a_{1}{ }^{\mathrm{id}}=\alpha_{1} x\left[\alpha_{1} x+\alpha_{2}(1-x)\right]^{2}\left[\frac{1+2 \alpha_{1}{ }^{0}}{a_{1}{ }^{0}\left(1+2 \alpha_{2}+\left(\alpha_{1}-\alpha_{2}\right) 2 x\right)}\right]^{3} \equiv K_{1}{ }^{\mathrm{IV}} \\
& a_{2}{ }^{\mathrm{id}}=\alpha_{2}(1-x)\left[\alpha_{2}(1-x)+\alpha_{1} x\right]^{2}\left[\frac{1+2 \alpha_{2}{ }^{0}}{\alpha_{2}{ }^{0}\left(1+2 \alpha_{2}+\left(\alpha_{1}-\alpha_{2}\right) 2 x\right)}\right]^{3} \equiv K_{2}{ }^{\mathrm{IV}} .
\end{aligned}
$$

Hier ergibt sich für die vollständige Dissoziation $\left(\alpha_{1}=\alpha_{1}^{0}=\alpha_{2}=\alpha_{2}{ }^{0}=1\right)$ :

$$
a_{1}{ }^{\mathrm{id}}=x, \quad a_{2}{ }^{\mathrm{id}}=(1-x) .
$$

Diese vier Beispiele zeigen, daß nur bei vollständiger Dissoziation im I. und IV. Fall, bei denen die Moleküle der beiden Komponenten je ein nicht gemeinsames Kation besitzen, die Aktivitäten $a_{1}$ bzw. $a_{2}$ mit dem Molenbruch $x$ bzw. $(1-x)$ identisch sind. In den anderen Fällen fI. und IV. bei unvollständiger Dissoziation und II. und III. bei vollständiger und unvollständiger Dissoziation) liegen kompliziertere Abhängigkeiten der Aktivitäten vom Molenbruch vor. Diese Tatsache hat bei der Beschreibung von Verdampfungsgleichgewichten, wie wir später sehen werden, eine wichtige Konsequenz.

\section{Aktivitätskoeffizienten}

Definiert man nach $\mathrm{HAASE}^{7}$ den temperatur-, druck- und konzentrationsabhängigen Aktivitätskoeffizienten $f_{i}$ der Komponente $i$ durch

$$
f_{i} \equiv a_{i} / a_{i}^{\text {id }} \quad(i=1,2),
$$

so gibt $f_{i}$ den Unterschied der Eigenschaften einer beliebigen Mischung zu den einer unvollständig dissoziierten idealen Mischung an, da für eine unvollständig dissoziierte ideale Mischung

$$
\begin{array}{lll} 
& a_{i}=a_{i}{ }^{\text {id }} & (i=1,2) \\
\text { ist und damit } & f_{i}=1 & (i=1,2)
\end{array}
$$

wird. $f_{i} \neq 1$ beschreibt also eine nichtideale Mischung mit beliebiger Dissoziation.

Die Aktivitätskoeffizienten sind nach (35) und (59) durch

$$
x\left[\frac{\partial \ln f_{1}}{\partial x}\right]_{T, P}+(1-x)\left[\frac{\partial \ln f_{2}}{\partial x}\right]_{T, P}=0
$$

miteinander verknüpft.

Für die reinen Komponenten 1 bzw. 2 erhält man durch den Grenzübergang $x \rightarrow 1 \quad\left(\alpha_{1} \rightarrow \alpha_{1}{ }^{0}\right)$ bzw. $x \rightarrow 0 \quad\left(\alpha_{2} \rightarrow \alpha_{2}{ }^{0}\right)$ aus (30a), (33), (44a) und (59) bzw. $(30 \mathrm{~b}),(33),(44 \mathrm{~b})$ und (59)

$$
\begin{array}{lll} 
& \lim _{x \rightarrow 0}\left(\ln f_{1}\right)=0, & \lim _{x \rightarrow 0} f_{1}=1 \\
\text { bzw. } & \lim _{x \rightarrow 0}\left(\ln f_{2}\right)=0, & \lim _{x \rightarrow 0} f_{2}=1
\end{array}
$$

in Analogie zu den Nichtelektrolytmischungen. 
Für die Aktivitätskoeffizienten $f_{1}$ und $f_{2}$ findet man aus (59) mit (44a) und (44 b) die recht komplizierten Ausdrücke

$$
f_{1}=a_{1} \frac{1}{\left[a_{1} x\right]^{\nu_{a}}\left[a_{1} x+\left(\nu^{*} / \nu_{b}\right) a_{2}(1-x)\right]^{\nu_{b}}}\left[\frac{\alpha_{1}{ }^{0}\left[1+\left(v_{2}-1\right) \alpha_{2}+\left(\left(v_{1}-1\right) a_{1}-\left(v_{2}-1\right) a_{2}\right) x\right]}{1+\left(v_{1}-1\right) a_{1}{ }^{0}}\right]^{v_{1}}
$$

und

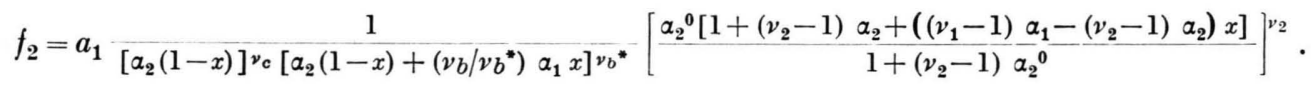

Bei den oben genannten vier Beispieltypen von Systemen I, II, III und IV ergibt sich nach (64a) und (64 b) die Beziehung

$$
\begin{gathered}
f_{i}=a_{i} / K_{i}{ }^{j}, \\
(i=1,2 ; j=\mathrm{I}, \mathrm{II}, \mathrm{III}, \mathrm{IV})
\end{gathered}
$$

und zwar für

I. (Typ $\mathrm{AB}+\mathrm{CB}$ ) bei Beachtung von (47) unter Verwendung der Abkürzung $K_{i}^{\mathrm{I}}(48 \mathrm{a})$ und $(48 \mathrm{~b})$,

II. (Typ $\mathrm{A}_{2} \mathrm{~B}_{2}+\mathrm{C}_{2} \mathrm{~B}_{2}$ ) bei Beachtung von (50) unter Verwendung der Abkürzung $K_{i}^{\mathrm{II}}(5 \mathrm{l}$ a) und $(51 \mathrm{~b})$,

III. (Typ $\mathrm{A}_{2} \mathrm{~B}+\mathrm{C}_{2} \mathrm{~B}$ ) bei Beachtung von (53) unter Verwendung der Abkürzung $K_{i}^{\text {III }}(54$ a) und (54 b) und

IV. (Typ $\mathrm{AB}_{2}+\mathrm{CB}_{2}$ ) bei Beachtung von (56) unter Verwendung der Abkürzung $K_{i}^{\text {IV }}$ (57 a) und (57 b).

Vollständige Dissoziation $\left(\alpha_{1}=\alpha_{1}{ }^{0}=\alpha_{2}=\alpha_{2}{ }^{0}=1\right)$ vereinfacht die Gl. (65) wesentlich. In den obigen Beispielen findet man nach (65) für die Aktivitätskoeffiizenten $f_{1}$ und $f_{2}$ bei

I. (Typ $\mathrm{AB}+\mathrm{CB})$ mit $(47)$, (48 a) und $(48 \mathrm{~b})$ :

$$
f_{1}=a_{1} / x, \quad f_{2}=a_{2} /(1-x), \quad(66 \mathrm{a}, \mathrm{b})
$$

II. (Typ $\left.\mathrm{A}_{2} \mathrm{~B}_{2}+\mathrm{C}_{2} \mathrm{~B}_{2}\right)$ mit (50), (51 a) und $(51 \mathrm{~b})$ :

$$
f_{1}=a_{1} / x^{2}, \quad f_{2}=a_{2} /(1-x)^{2}, \quad(67 \mathrm{a}, \mathrm{b})
$$

III. (Typ $\left.\mathrm{A}_{2} \mathrm{~B}+\mathrm{C}_{2} \mathrm{~B}\right)$ mit (53), (54 a) und $(54 \mathrm{~b})$ :

$$
f_{1}=a_{1} / x^{2}, \quad f_{2}=a_{2} /(1-x)^{2}, \quad(68 \mathrm{a}, \mathrm{b})
$$

und

IV. (Typ $\mathrm{AB}_{2}+\mathrm{CB}_{2}$ ) mit (56), (57 a) und $(57 \mathrm{~b})$ :

$$
f_{1}=a_{1} / x, \quad f_{2}=a_{2} /(1-x) . \quad(69 \mathrm{a}, \mathrm{b})
$$

Man erkennt, daß im Falle vollständiger Dissoziation einfache Ausdrücke für die Aktivitätskoeffizienten $f_{1}$ und $f_{2}$ erzielt werden. Nur die Beispiele I (Typ $\mathrm{AB}+\mathrm{CB}$ : zwei Molekülarten mit je einem Kation und einem gemeinsamen Anion) und IV (Typ $\mathrm{AB}_{2}+\mathrm{CB}_{2}$ : zwei Molekülarten mit je einem Kation und zwei gemeinsamen Anionen) liefern bei vollständiger Dissoziation für die Aktivitätskoeffizienten Ausdrücke, die mit denen für Nichtelektrolytmischungen übereinstimmen.

\section{Verdampfungsgleichgewicht}

Liegt ein System aus einer flüssigen Mischung der (teilweise dissoziierten) Komponenten 1 und 2 und dem koexistierenden Dampf vor, lautet die allgemeine Gleichgewichtsbedingung ${ }^{2}$ :

$$
\mu_{i}(T, p, x)=\mu_{i}^{G}\left(T, p, x^{\mathrm{G}}\right) \quad(i=1,2) .
$$

Für das Gleichgewicht zwischen Flüssigkeit und Dampf des reinen Stoffes $i$ gilt entsprechend:

$$
\mu_{0 i}\left(T, p_{0 i}\right)=\mu_{0 i}^{\mathrm{G}}\left(T, p_{0 i}\right) \quad(i=1,2) .
$$

$\mu_{i}$ bzw. $\mu_{i}^{\mathrm{G}}$ bzw. $\mu_{0 i}$ bzw. $\mu_{0 i}^{\mathrm{G}}$ ist das chemische Potential der Komponente $i$ in der flüssigen Mischung bzw. im mit dieser Mischung koexistierenden Dampf bzw. des reinen flüssigen Stoffes $i$ bzw. im Gleichgewichtsdampf des reinen Stoffes $i$.

Das chemische Potential der Komponente $i$ in der flüssigen Mischphase $\mu_{i}$ hängt von der Temperatur $T$, vom Druck $p$ und von der Zusammensetzung $x$ der Flüssigkeit, das des Stoffes $i$ in der gasförmigen Mischung $\mu_{i}^{G}$ von der Temperatur $T$, vom Druck $p$ und von der Zusammensetzung $x^{\mathrm{G}}$ der Gasphase ab. Im Gleichgewicht sind von den vier Zustandsvariablen des Systems aus den beiden Phasen $\left(T, p, x, x^{\mathrm{G}}\right)$ zwei voneinander unabhängig (zum Beispiel $T, x$ ). Die beiden anderen liegen auf Grund der Phasenregel fest (zum Beispiel $p, x^{\mathrm{G}}$ ).

Bei der reinen Komponente $i$ hängt das chemische Potential dieses Stoffes für die Flüssigkeit $\mu_{0 i}$ und den Dampf $\mu_{0 i}^{G}$ von der Temperatur $T$ und vom Druck $p_{0 i}$ ab. Im Gleichgewicht zwischen Flüssigkeit und Dampf ist eine Variable (zum Beispiel T) frei wählbar, die andere (zum Beispiel $p_{0 i}$ ) stellt sich ein. Die durch die Gl. (33) eingeführte Aktivität $a_{i}$ der Komponente $i$ wird für die Temperatur $T$ und die Zusammensetzung $x$ auf einen Standarddruck $P_{0}$ bezogen, der gewöhnlich einen Wert von $P_{0}=1 \mathrm{~atm}$ hat. Aus (33) folgt dann:

$R T \ln a_{i}=\mu_{i}\left(T, P_{0}, x\right)-\mu_{0 i}\left(T, P_{0}\right) \quad(i=1,2)$. 
Die mit der Druckänderung vom Standarddruck $P_{0}$ auf den Gleichgewichtsdruck $p$ verbundene Änderung des chemischen Potentials der Komponente $i$ in der flüssigen Mischphase bei fester Temperatur $T$ und konstanter Zusammensetzung $x$ wird durch ${ }^{2}$

$\mu_{i}\left(T, P_{0}, x\right)=\mu_{i}(T, p, x)-\int_{P_{0}}^{p} V_{i} \mathrm{~d} P(i=1,2)$

und die mit der Druckänderung vom Standarddruck
$P_{0}$ auf den Gleichgewichtsdruck $p_{0 i}$ verknüpfte Änderung des chemischen Potentials der Komponente $i$ in der reinen Flüssigkeit bei fester Temperatur $T$ durch

$\mu_{0 i}\left(T, P_{0}\right)=\mu_{0 i}\left(T, p_{0 i}\right)-\int_{P_{0}}^{p_{0 i}} V_{0 i} \mathrm{~d} P(i=1,2)$

gegeben, wobei $V_{i}$ das partielle molare Volumen der flüssigen Komponente $i$ und $V_{0 i}$ das molare Volumen der reinen Flüssigkeit $i$ ist.

Einsetzen von (73 a) und (73 b) in (72) ergibt:

$$
R T \ln a_{i}=\mu_{i}(T, p, x)-\mu_{0 i}\left(T, p_{0 i}\right)+\int_{P_{0}}^{p_{0 i}} V_{0 i} \mathrm{~d} P-\int_{P_{0}}^{p} V_{i} \mathrm{~d} P(i=1,2),
$$

woraus unter Verwendung der Gleichgewichtsbedingungen (70) und (71) folgt:

$$
R T \ln a_{i}=\mu_{i}^{G}\left(T, p, x^{\mathrm{G}}\right)-\mu_{0 i}^{\mathrm{G}}\left(T, p_{0 i}\right)+\int_{P_{0}}^{p_{0 i}} V_{0 i} \mathrm{~d} P-\int_{P_{0}}^{p} V_{i} \mathrm{~d} P(i=1,2) .
$$

Für das chemische Potential $\mu_{i}^{\mathrm{G}}$ des gasförmigen Stoffes $i$ in einer Gasmischung mit dem Druck $p$ und das chemische Potential $\mu_{0 i}^{\mathrm{G}}$ des reinen Gases $i$ beim Druck $p_{0 i}$ gilt allgemein ${ }^{2}$ :

$$
\begin{aligned}
& \mu_{i}^{\mathrm{G}}\left(T, p, x^{\mathrm{G}}\right)=R T \ln \frac{p x^{\mathrm{G}}}{P^{\dagger}}+\int_{0}^{p}\left(V_{i}^{\mathrm{G}}-\frac{R T}{P}\right) \mathrm{d} P, \\
& \mu_{0 i}^{\mathrm{G}}\left(T, p_{0 i}\right)=R T \ln \frac{p_{0 i} x_{0}^{\mathrm{G}}}{P^{\dagger}}+\int_{0}^{p_{0 i}}\left(V_{0 i}^{\mathrm{G}}-\frac{R T}{P}\right) \mathrm{d} P \quad \text { mit } \quad P^{\dagger} \equiv 1 \mathrm{~atm} .
\end{aligned}
$$

Hierin bedeutet $x_{i}^{\mathrm{G}}$ bzw. $x_{0 i}^{\mathrm{G}}$ den Molenbruch der Komponente $i$ im Dampf der flüssigen Mischphase bzw. im Dampf der reinen flüssigen Komponente $i$ und $V_{i}^{\mathrm{G}}$ bzw. $V_{0 i}^{\mathrm{G}}$ das partielle molare Volumen der Komponente $i$ im Gleichgewichtsdampf der flüssigen Mischphase bzw. das molare Volumen des Dampfes der reinen Komponente $i$. Tritt im reinen Dampf weder Dissoziation noch Assoziation auf, ist

$$
x_{0 i}^{\mathrm{G}}=1 \text {. }
$$

Die in den Beziehungen (76a) und $(76 \mathrm{~b})$ auftretenden Integrale berücksichtigen das nichtideale Verhalten des Dampfes der Mischung und der reinen Komponenten.

Werden die Gln. (76a) und (76b) in (75) eingeführt, erhält man :

$$
\begin{aligned}
R T \ln a_{i} & =R T \ln \frac{p x_{i}^{G}}{p_{0 i} x_{0 i}^{G}}+R_{i}^{G}+R_{i}, \\
a_{i} & =\frac{p x_{i}^{G}}{p_{0 i}^{G} x_{0 i}^{G}} \exp \left\{\frac{R_{i}^{G}+R_{i}}{R T}\right\},
\end{aligned}
$$

wobei die Abkürzungen

$$
\begin{gathered}
R_{i}^{\mathrm{G}} \equiv \int_{0}^{p}\left(V_{i}^{\mathrm{G}}-\frac{R T}{P}\right) \mathrm{d} P-\int_{0}^{p_{0 i}}\left(V_{0 i}^{\mathrm{G}}-\frac{R T}{P}\right) \mathrm{d} P \\
\text { und } \quad R_{i} \equiv \int_{P_{0}}^{p_{0 i}} V_{0 i} \mathrm{~d} P-\int_{P_{0}}^{p} V_{i} \mathrm{~d} P
\end{gathered}
$$

benutzt wurden.

Bei Systemen mit vernachlässigbarer Druckabhängigkeit des chemischen Potentials der flüssigen Phase $\left(R_{i}=0\right)$, idealer Dampfphase $\left(R_{i}^{G}=0\right)$ ohne Dissoziation oder Assoziation der Moleküle im reinen Dampf $\left(x_{0 i}^{\mathrm{G}}=1\right)$, folgt aus (77) die einfache Be. ziehung:

$$
a_{i}=p_{i} / p_{0 i},
$$

wobei $p_{i}$ der durch

$$
p_{i} \equiv x_{i}^{G} p
$$

definierte Partialdruck der Komponente $i$ im Dampf ist. Für die Aktivitätskoeffizienten $f_{1}$ und $f_{2}$ erhält man aus (64 a) und (77) mit $i=1$ bzw. aus $(64 \mathrm{~b})$ und (77) mit $i=2$ : 


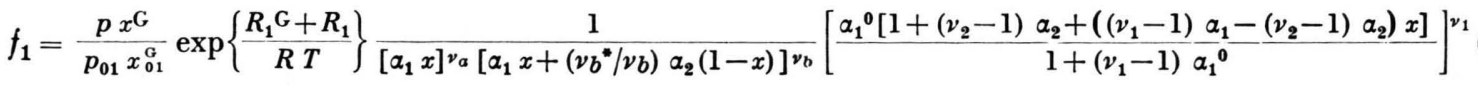

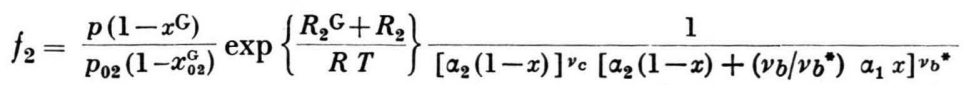

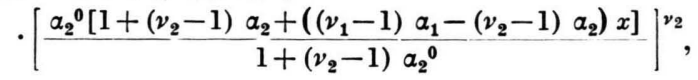

worin für $i=1$ bzw. $i=2$ gesetzt ist:

$$
x_{1}=x, \quad x_{2}=(1-x) \text {. }
$$

Aus den Daten für den Gleichgewichtsdampf der Mischphase (Dampfdruck $p$, Molenbruch $x^{\mathrm{G}}$ und partieles molares Volumen $\left.V_{i}^{G}\right)$, für den Dampf der reinen Komponente $i$ (Dampfdruck $p_{0 i}$, Molenbruch $x_{0 i}^{\mathrm{G}}$, und molares Volumen $V_{0 i}^{\mathrm{G}}$, , für die flüssige $\mathrm{Mi}$. schung (Molenbruch $x$, partielles molares Volumen $V_{i}$ und Dissoziationsgrad $\alpha_{i}$ ) und für die reinen flüsigen Stoffe $i$ (partielles molares Volumen $V_{0 i}$ und Dissoziationsgrad $\alpha_{i}$ ) sowie den Zerfallszahlen $v_{a}, v_{b}, v_{c}$ und $v_{b}^{*}$ lassen sich unter Verwendung von (9) und (10) die Aktivitätskoeffizienten unseres Systems nach $(81 \mathrm{a})$ und $(81 \mathrm{~b})$ für jede Temperatur $T$ und Zusammensetzung $x$ der flüssigen Phase berechnen. Tritt in der Dampfphase Assoziation auf, wie es z. B. bei den aliphatischen Monocarbonsäuren der Fall ist, so kann bei Verwendung der zur Untersuchung von Verdampfungsgleichgewichten üblichen Umlaufapparaturen die Bestimmung der Molenbrüche $x^{\mathrm{G}}$ bzw. $x_{0 i}^{\mathrm{G}}$ durch Analyse einer Probe des kondensierten Dampfes nur bei Kenntnis der Assoziationskonstanten des Dampfgemisches bzw. des reinen Dampfes erfolgen.

Bei Gültigkeit der Voraussetzungen zur Gl. (79) (keine Druckabhängigkeit der chemischen Potentiale der Komponenten in der flüssigen Mischung: $R_{i}=0$; ideale Gasphase: $R_{i}^{G}=0$; keine Dissoziation oder Assoziation im Dampf: $x_{0 i}^{\mathrm{G}}=1$ ) vereinfachen sich (81 a) und (81 b) bei Beachtung von (80) zu

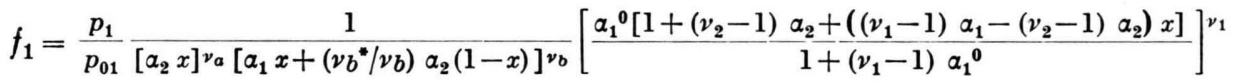

$$
\begin{aligned}
& f_{2}=\frac{p_{2}}{p_{02}} \frac{1}{\left[a_{2}(1-x)\right]^{\nu_{c}}\left[a_{2}(1-x)+\left(v_{b} / \nu_{b}^{*}\right) a_{1} x\right]^{\nu_{b}^{*}}}\left[\frac{a_{2}{ }^{0}\left[1+\left(v_{2}-1\right) a_{2}+\left(\left(v_{1}-1\right) a_{1}-\left(v_{2}-1\right) a_{2}\right) x\right]}{1+\left(v_{2}-1\right) a_{2}{ }^{0}}\right]^{\nu_{2}} \text {. }
\end{aligned}
$$

Für die früher erörterten Beispiele I (Typ AB $+\mathrm{CB})$, II (Typ $\mathrm{A}_{2} \mathrm{~B}_{2}+\mathrm{C}_{2} \mathrm{~B}_{2}$ ), III (Typ $\mathrm{A}_{2} \mathrm{~B}+\mathrm{C}_{2} \mathrm{~B}$ ) und IV (Typ $\mathrm{AB}_{2}+\mathrm{CB}_{2}$ ) erhält man unter Benutzung der obigen Annahmen aus (82 a) und (82 b)

$f_{i}=\frac{p_{i}}{p_{0 i}} \frac{1}{K_{i}{ }^{i}} \quad(i=1,2 ; j=\mathrm{I}, \mathrm{II}, \mathrm{III}, \mathrm{IV})$,

wobei im Sonderfall I (48 a) und (48b), im Sonderfall II (51 a) und $(51 \mathrm{~b})$, im Sonderfall III (54 a) und $(54 \mathrm{~b})$ und im Sonderfall IV (57 a) und $(57 \mathrm{~b})$ benutzt wurden. Liegt vollständige Dissoziation vor $\left(\alpha_{1}=\alpha_{1}^{0}=\alpha_{2}=\alpha_{2}{ }^{0}=1\right)$, resultiert bei I bzw. IV aus (66a), (66 b) und (79) bzw. aus $(69 a),(69 b)$ und (79)

$f_{1}=p_{1} / p_{01} x$, bzw. $f_{2}=p_{2} / p_{02}(1-x), \quad(84 \mathrm{a}, \mathrm{b})$ während man bei II bzw. III aus $(67 \mathrm{a}),(67 \mathrm{~b})$ und (79) bzw. (68a), (68 b) und (79)

$f_{1}=p_{1} / p_{01} x^{2} \quad$ bzw. $\quad f_{2}=p_{2} / p_{02}(1-x)^{2} \quad(85 \mathrm{a}, \mathrm{b})$ erhält.

Nur bei der idealen vollständig dissoziierten flüssigen Mischung, bei der die chemischen Potentiale der Komponenten nicht vom Druck abhängen und dessen Gleichgewichtsdampf ein ideales Gas und weder assoziiert noch dissoziiert ist, ergibt sich aus (84 a) und (84 b) mit (61) das Raoultsche Gesetz:

$$
p_{1}=x p_{01} \quad \text { und } \quad p_{2}=(1-x) p_{02} \quad(86 \mathrm{a}, \mathrm{b})
$$

für den gesamten Konzentrationsbereich.

\section{Zusammenfassung}

Es wird eine allgemeine thermodynamische Beschreibung eines Systems aus zwei Komponenten angegeben, die in einer Stufe unvollständig dissoziieren, wobei eine Ionenart gemeinsam ist. Die Grenzfälle „vollständige Dissoziation" und „keine Dissoziation" werden in der systematischen Betrachtung gleichzeitig mit erfaßt. Die ideale unvollständig dissoziierte Mischung wird definiert, wobei als Konsequenz dieser Definition die häufig unbekannten Dissoziationsgrade der Komponenten in der Mischung und im reinen Zustand zur Beschreibung erforderlich sind. Exakte Formeln zur Berechnung 
der Aktivitätskoeffizienten der beiden Komponenten aus experimentellen Daten werden hergeleitet. Für praktische Auswertungen werden einige sinnvolle Näherungen der recht komplizierten Beziehungen durchgeführt. Man erhält das Raoultsche Gesetz für den gesamten Konzentrationsbereich nur im Grenzfall vollständiger Dissoziation bei Systemen, deren
Komponenten pro Molekül nur ein nicht gemeinsames Ion besitzen. Auswertungen von Literaturdaten und eigene Messungen sollen in einer späteren Arbeit mitgeteilt werden.

Herrn Prof. Dr. R. HAase danke ich für wertvolle Anregungen.

\title{
Thermoosmose in Flüssigkeiten
}

\author{
IV. Weitere Messungen \\ R. HAASE, H. J. DE GreifF und H.-J. BuchneR \\ Institut für Physikalische Chemie der Rheinisch-Westfälischen Technischen Hochschule Aachen \\ (Z. Naturforsch. 25 a, 1080-1085 [1970]; eingegangen am 29. April 1970)

\begin{abstract}
Neue Messungen am System Cellophan (600) + Wasser im Temperaturbereich zwischen $10^{\circ} \mathrm{C}$ und $90^{\circ} \mathrm{C}$ werden mitgeteilt und diskutiert. Es handelt sich um die Permeabilität, die Uberführungswärme und die thermoosmotische Permeabilität, die für Permeation und Thermoosmose maßgebend sind, sowie um die Temperaturabhängigkeit des Wassergehaltes der Folie bei Quellungs. gleichgewicht. Es wird untersucht, ob Cellophanfolien in flüssigem Wasser als Porenmembranen bzw. als Löslichkeitsmembranen anzusehen sind. Besondere Aufmerksamkeit wird der Richtungsumkehr der Thermoosmose bei einer mittleren Temperatur von etwa $56^{\circ} \mathrm{C}$ gewidmet.
\end{abstract}

\section{Einleitung}

Im Anschluß an unsere früheren Untersuchungen über Permeation und Thermoosmose ${ }^{1-4}$, deren experimenteller Teil ${ }^{2,3}$ sich in erster Linie auf das System Cellophan + Wasser bezog, teilen wir jetzt die Ergebnisse weiterer Messungen an diesem System mit. Die Versuche erstrecken sich auf den Tempera. turbereich zwischen etwa $10^{\circ} \mathrm{C}$ und rund $90{ }^{\circ} \mathrm{C}$. Auch gehen wir in diesem Zusammenhang auf Messungen des Quellungsgleichgewichtes in Abhängigkeit von der Temperatur sowie auf die Interpretation der Permeation und Thermoosmose in Flüssigkeiten ein.

\section{Permeabilität, Überführungswärme und thermoosmotische Permeabilität}

Unsere Versuche betreffen zunächst die Permeation und Thermoosmose von reinem Wasser durch Cellulosemembranen bei verschiedenen (mittleren) Temperaturen. Die Membranen sind Cellophanfolien mit der Fabrikationsbezeichnung 600 der Fa. Kalle

Sonderdruckanforderungen an Prof. Dr. R. HAASE, Lehrstuhl für Physikalische Chemie II der Technischen Hochschule Aachen, D-5100 Aachen, Templergraben 59.

1 R. HaAse, Z. Physik. Chem. N. F. 21, 244 [1959].
AG., Wiesbaden-Biebrich. Die Experimente verlaufen nach dem bereits angegebenen Verfahren ${ }^{3}$. Die Ergebnisse der isothermen Versuche sind prak. tisch neu. Die Resultate der nicht-isothermen Experimente stellen eine Ergänzung und Korrektur der früheren Ergebnisse dar, da die Meßgenauigkeit gesteigert werden konnte und ein größerer Temperaturbereich erfaßt wurde. Alle Messungen sind an derselben Membran durchgeführt worden.

Die Permeabilität $A$ wird, wie früher ${ }^{3}$ ausführlich dargelegt, aus dem zeitlichen Verlauf eines Versuches im isothermen System ermittelt. Die gefundenen Wert von $A$ (SI-Einheit: $\mathrm{mol} \mathrm{kg}^{-1} \mathrm{~s}$ ) sind in Tab. 1 aufgeführt und in Abb. 1 graphisch dargestellt. Wie man erkennt, ist $A$ stets positiv, wie es sein muß, und nimmt mit wachsender Temperatur zu. (Der Wert bei $60,55{ }^{\circ} \mathrm{C}$ oder derjenige bei $65,47^{\circ} \mathrm{C}$ dürfte auf Meßfehlern beruhen.)

Die Überführungswärme $Q^{*}$ wird, wie ebenfalls schon beschrieben ${ }^{3}$, aus der stationären Druckdifferenz bei nichtisothermen Systemen gewonnen. Die Werte von $Q^{*}$ (SI-Einheit: $\mathrm{J} \mathrm{mol}^{-1}$ ) sind aus Tab. 1

2 R. Haase u. C. Steinert, Z. Physik. Chem. N. F. 21, 270 [1959].

3 R. HaAse u. H. J. de Greiff, Z. Physik. Chem. N. F. 44, 301 [1965].

${ }^{4}$ R. HaAse, Z. Physik. Chem. N. F. 51, 315 [1966]. 